Proceedings

\title{
Thermosensitive Nasal In Situ Gels of Lipid-Based Nanosystems to Improve the Treatment of Alzheimer's Disease ${ }^{\dagger}$
}

\author{
Sara Cunha ${ }^{1, *}$, Ben Forbes ${ }^{2}$, José Manuel Sousa Lobo ${ }^{1}$ and Ana Catarina Silva ${ }^{1,3}$ \\ 1 UCIBIO/REQUIMTE, MEDTECH Laboratory of Pharmaceutical Technology, Department of Drug Sciences, \\ Faculty of Pharmacy, University of Porto, 4050-313 Porto, Portugal; slobo@ff.up.pt (J.M.S.L.); \\ ana.silva@ff.up.pt (A.C.S.) \\ 2 Faculty of Life Sciences and Medicine, Institute of Pharmaceutical Science, King's College London, \\ London SE1 9NH, UK; ben.forbes@kcl.ac.uk \\ 3 UFP Energy, Environment and Health Research Unit (FP ENAS), Fernando Pessoa University, \\ 4249-004 Porto, Portugal \\ * Correspondence: up201510339@ff.up.pt; Tel.: +351-22-042-8500 \\ + Presented at the 1st International Electronic Conference on Pharmaceutics, 1-15 December 2020; \\ Available online: https://iecp2020.sciforum.net/.
}

Citation: Cunha, S.; Forbes, B.; Lobo, J.M.S.; Silva, A.C. Thermosensitive Nasal In Situ Gels of Lipid-Based Nanosystems to Improve the Treatment of Alzheimer's Disease. Proceedings 2021, 78, 37. https://doi. org/10.3390/IECP2020-08648

Published: 1 December 2020

Publisher's Note: MDPI stays neutral with regard to jurisdictional claims in published maps and institutional affiliations.

\section{(i)}

Copyright: (C 2020 by the authors. Licensee MDPI, Basel, Switzerland. This article is an open access article distributed under the terms and conditions of the Creative Commons Attribution (CC BY) license (http://creativecommons.org/licenses/by/4.0/).

\begin{abstract}
Thermosensitive in situ gels are promising formulations for the management of Alzheimer's disease (AD), since they increase the residence time of lipid-based nanosystems in the nasal cavity, improving drug therapeutic efficacy. The purpose of this study is to prepare thermosensitive in situ gels with anticholinesterase inhibitor (RVG)-loaded nanostructured lipid carriers (NLC) and nanoemulsions to improve the residence time of the formulations in the nasal cavity. Different concentrations of thermosensitive polymers were added to the RVG-loaded NLC and to the RVGloaded nanoemulsion to optimize the gelation temperature of the in situ gels; concentrations of $17 \%$ $(\%, w / w)$ of Kolliphor ${ }^{\circledR}$ P407 and $0.3 \%(\%, w / w)$ of Methocel $^{\mathrm{TM}}$ K4M were selected. The in situ gels of the RVG-loaded NLC and RVG-loaded nanoemulsion had a particle size, PDI, ZP, and pH of, respectively: $141.70 \pm 0.40 \mathrm{~nm}$ and $146.10 \pm 1.73 \mathrm{~nm} ; 0.45 \pm 0.00$ and $0.43 \pm 0.02 ;-4.06 \pm 1.03 \mathrm{mV}$ and $-4.09 \pm 0.71 \mathrm{mV}, 6.60 \pm 0.01$ and $7.00 \pm 0.02$. In addition, these in situ gels showed a non-Newtonian plastic behavior, and the texture parameters presented desirable values for nasal administration. From these results, we concluded that the developed in situ gels can be used to improve the treatment of $\mathrm{AD}$ through the nose-to-brain route.
\end{abstract}

Keywords: Alzheimer's disease; nose-to-brain delivery; lipid-based nanosystems; thermosensitive in situ gels

\section{Introduction}

Alzheimer's disease (AD) is a neurodegenerative disorder associated with neuronal degeneration and loss of cognitive functions, which lead to severe dementia. Currently, there is no fully effective treatment for AD; drugs are administered in conventional pharmaceutical dosage forms, which reduce the symptoms of the disease while maintaining the patient's quality of life [1,2]. Examples of drugs used in the management of AD include the acetylcholinesterase inhibitors (AChEs), which increase the acetylcholine levels by inhibiting the acetylcholinesterase activity. However, these drugs are generally administered orally and absorbed into the systemic circulation, where they undergo enzymatic and metabolic degradation which decreases their therapeutic effectiveness. In addition, drugs need to cross the blood-brain-barrier (BBB) to reach the central nervous system (CNS), which is challenging [3,4]. Thereby, alternative routes to deliver AChEs inhibitors have been investigated, including nose-to-brain delivery, which allows the direct passage 
of the drugs from the nasal cavity to the brain through the olfactory and trigeminal nerves. However, the physiological mechanism of mucociliary clearance is a significant barrier to the absorption of drugs in the nasal cavity, which are quickly eliminated [5-7]. To circumvent this limitation, the use of mucoadhesive formulations can improve the drugs' residence time in the nasal cavity through the formation of electrostatic interactions between the formulation and mucin [8]. Thermosensitive polymers, such as poloxamers, and mucoadhesive agents, such as chitosan and hydroxypropyl methylcellulose, have been successfully used to prepare thermosensitive in situ nasal gels [9].

To maximize their mucoadhesive properties, in situ gels have been associated with lipid-based nanosystems. Among these, nanostructured lipid carriers (NLC) and nanoemulsions have proved to be ideal candidates for nasal drug delivery due to their physicochemical properties. The combination of in situ gels with lipid-based nanosystems is useful for the transport of lipophilic drugs, since they are encapsulated in the lipid matrix of the nanosystem within the gel structure, which provides the drugs with high protection and a prolonged release [10,11].

This work aimed to develop thermosensitive in situ nasal gels of NLC and nanoemulsions loaded with an acetylcholinesterase inhibitor (RVG) to improve residence time in the nasal cavity.

\section{Experiments}

\subsection{Materials}

Precirol $^{\circledR}$ ATO 5 (glyceryl distearate/glyceryl palmitostearate) was a gift from Gattefossé (Lyon, France), Miglyol ${ }^{\circledR} 812$ (triglycerides of capric/caprylic acids), alpha-tocopherol acetate (vitamin E), polysorbate 80 (Tween ${ }^{\circledR} 80$ ) and glycerin were acquired from Acofarma (Barcelona, Spain), phospholipon ${ }^{\circledR}$ 90G (phosphatidylcholine, hydrogenated) was donated from Lipoid (Ludwigshafen am Rhein, Germany), and benzalkonium chloride was acquired from Acef (Piacenza, Italy). Kolliphor ${ }^{\circledR}$ P407 (poloxamer 407) and Methocel $^{\mathrm{TM}} \mathrm{K} 4 \mathrm{M}$ (hydroxypropyl methylcellulose) were donated from Basf (Porto, Portugal) and Coloron ${ }^{\circledR}$ (Bay City, United States), respectively. The water used in all experiments was purified and obtained from a Milli ${ }^{\circledR}$ P Plus, Millipore ${ }^{\circledR}$ (Darmstadt, Germany).

\subsection{Preparation of RVG-Loaded NLC and RVG-Loaded Nanoemulsions}

RVG-loaded NLC was previously optimized by Cunha et al. [12] using the high-pressure homogenization method (HPH) and the RVG-loaded nanoemulsions were prepared by the ultrasound technique. Briefly, the lipid phase and the aqueous phase were heated at $70^{\circ} \mathrm{C}$. When both phases were at the same temperature, the aqueous phase was added to the lipid phase. Subsequently, the mixture was emulsified through high-speed stirring with an Ultra-Turrax ${ }^{\circledR} \mathrm{T} 25$ at 13,400 rpm for $5 \mathrm{~min}$. The oil-in-water (O/W) emulsion obtained was sonicated by means of an VCX130 ultrasonic processor, with a power output amplitude of $85 \%$ for $15 \mathrm{~min}$. The hot $\mathrm{O} / \mathrm{W}$ nanoemulsion was transferred to glass vials and cooled to room temperature $\left(20.0 \pm 0.5^{\circ} \mathrm{C}\right)$. A drug concentration (RVG) of $0.12 \%(\%$, $w / w)$ was added to the lipid phase.

\subsection{Screening of Excipients}

Kolliphor $^{\circledR}$ P407 (poloxamer 407) was used as gelling agent, while Methocel ${ }^{\mathrm{TM}} \mathrm{K} 4 \mathrm{M}$ (hydroxypropyl methylcellulose) was used as mucoadhesive agent. The suitable sol-gel transition temperature for nasal application ranges from 28 to $37^{\circ} \mathrm{C}$ [13]. Different concentrations of Kolliphor ${ }^{\circledR}$ P407 $(9,12,17$, and 20, \%, w/w) were added to the NLC dispersion and to the nanoemulsion, and the occurrence of gelation was analyzed after $24 \mathrm{~h}$ of storage at $34.4 \pm 0.5^{\circ} \mathrm{C}$. After selecting the most suitable concentration of Kolliphor ${ }^{\circledast} \mathrm{P} 407$, $0.3 \%$ and $0.5 \%(\%, w / w)$ of Methocel $^{\mathrm{TM}} \mathrm{K} 4 \mathrm{M}$ were added to the in situ gels according to a previous work developed by Gadhave et al. [11]. 


\subsection{Preparation of Thermosensitive In Situ Gels}

In situ gels of RVG-loaded NLC and RVG-loaded nanoemulsions were prepared using the cold technique described by Fatouh et al. and Almeida et al. [14,15]. Briefly, the Kolliphor ${ }^{\circledast}$ P407 was added to the RVG-loaded NLC dispersion and to the RVG-loaded nanoemulsion and stirred at $743 \mathrm{rpm}$, at $4.0 \pm 0.5^{\circ} \mathrm{C}$. The formed gels were further stored at $4.0 \pm 0.5^{\circ} \mathrm{C}$ to eliminate the air incorporated during the preparation. Afterwards, Metho$\mathrm{cel}^{\mathrm{TM}} \mathrm{K} 4 \mathrm{M}$ was added to the formulations with a mechanical stirrer at $743 \mathrm{rpm}$. The final formulations were stored at $4.0 \pm 0.5^{\circ} \mathrm{C}$ for $24 \mathrm{~h}$ and then incubated at $34.4 \pm 0.5{ }^{\circ} \mathrm{C}$ for 24 h.

An in situ control gel was also prepared according to the cold method. Briefly, $0.12 \%$ $(\%, w / w)$ of RVG, $0.02 \%(\%, w / w)$ of benzalkonium chloride, $1.70 \%(\%, w / w)$ of glycerin, and $17 \%(\%, w / w)$ of Kolliphor ${ }^{\circledR} \mathrm{P} 407$ were dispersed in $100 \mathrm{~mL}$ of purified water at $4.0 \pm$ $0.5^{\circ} \mathrm{C}$ under magnetic stirring. Then, $0.3 \%(\%, w / w)$ of Methocel $^{\mathrm{TM}} \mathrm{K} 4 \mathrm{M}$ was added to the previously prepared gel and mixed with the magnetic stirrer. The prepared formulation was stored in a refrigerator at $4.0 \pm 0.5^{\circ} \mathrm{C}$ for $48 \mathrm{~h}$ and then incubated at $34.4 \pm 0.5^{\circ} \mathrm{C}$ for $24 \mathrm{~h}$.

\subsection{Particle Size, Polydispersity Index (PDI), and Zeta Potential (ZP)}

For the determination of particle/droplet size and PDI, the RVG-loaded NLC and RVG-loaded nanoemulsion and their respective in situ gels were previously diluted in ultrapure water in a proportion of 1:100. The measurements were performed by a dynamic light scattering technique (DLS) using a Malvern Nano-Zetasizer (Malvern, UK). ZP measurements were performed by laser doppler electrophoresis using the same apparatus. The temperature was set at $25.0 \pm 0.5^{\circ} \mathrm{C}$. Each sample was analyzed in triplicate $(n=3)$, and the results were reported as the mean \pm standard deviation (SD).

\subsection{Organoleptic Analysis}

The general macroscopic appearance of the in situ gels was analyzed $48 \mathrm{~h}$ after the production, at $20.0 \pm 0.5^{\circ} \mathrm{C}$.

\section{7. $\mathrm{pH}$ and Osmolarity}

The $\mathrm{pH}$ was measured using a BASIS 20 calibrated digital $\mathrm{pH}$ meter (Crison Instruments, s.a., Barcelona, Spain), and the osmolarity with a Type 6 osmometer (Löser Messtechnik, Berlin-Spandau, Germany). The values were adjusted to the nasal physiological values, using sodium hydroxide for $\mathrm{pH}$ (4.5-6.5) and glycerin for osmolarity (290 $\mathrm{mOsm} / \mathrm{kg})$.

\subsection{Rheological and Texture Analysis}

Rheological experiments were conducted in the in situ gels of the RVG-loaded NLC, RVG-loaded nanoemulsion, and in situ control gel with RVG, using a rotational viscometer (Haake VT-550 type Searle (Haake Viscotester ${ }^{\mathrm{TM}}$ 550; Thermo Fisher Scientific, Waltham, MA, USA) with a coaxial cylinder sensor (Rotor SV DIN 53019/ISO 3219), at $20.5 \pm$ $0.5^{\circ} \mathrm{C}$ and $34.4 \pm 0.5^{\circ} \mathrm{C}$. The measurements were performed in triplicate $(n=3)$, and the results were reported as the mean $\pm \mathrm{SD}$. The formulations' mechanical properties of adhesiveness and firmness were evaluated through a texture analysis. A texture analyzer (TAXT2i; Stable Micro Systems, Godalming, UK) with a load cell of $5 \mathrm{~kg}$, a trigger force of $0.049 \mathrm{~N}$, a $0.5 \mathrm{~mm}$ diameter cylindrical probe, a penetration depth of $5 \mathrm{~mm}$, and a test speed of $3 \mathrm{~mm} \cdot \mathrm{s}^{-1}$ was used. The experiments were performed in triplicate $(n=3)$, and the results were reported as the mean $\pm S D$, at $20.5 \pm 0.5^{\circ} \mathrm{C}$. 


\section{Results}

\subsection{Screening of Excipients}

The concentrations of Kolliphor ${ }^{\circledR} \mathrm{P} 407$ that allowed the sol-gel transition at $34.4 \pm 0.5$ ${ }^{\circ} \mathrm{C}$ was $17 \%(w / w)$. According to a previous work developed by Gadhave et al., the Methocel $^{\mathrm{TM}} \mathrm{K} 4 \mathrm{M}$ concentration selected was $0.3 \%(\%, w / w)[11]$.

\subsection{Particle Size, PDI and ZP}

Table 1 shows the results of the particle size, PDI, and ZP of the RVG-loaded NLC and RVG-loaded nanoemulsions and their respective in situ gels.

Table 1. Results of particle/droplet size, polydispersity index (PDI), and zeta potential (ZP).

\begin{tabular}{|c|c|c|c|}
\hline & Size (nm) & PDI & $\mathrm{ZP}(\mathrm{mV})$ \\
\hline NLC $^{1}$ & $114.00 \pm 1.91$ & $0.22 \pm 0.00$ & $-30.63 \pm 0.29$ \\
\hline NanoE 2 & $135.80 \pm 0.50$ & $0.14 \pm 0.00$ & $-20.87 \pm 0.21$ \\
\hline In situ NLC gel ${ }^{3}$ & $141.70 \pm 0.40$ & $0.45 \pm 0.00$ & $-4.06 \pm 1.03$ \\
\hline In situ NanoE gel 4 & $146.10 \pm 1.73$ & $0.43 \pm 0.02$ & $-4.09 \pm 0.71$ \\
\hline
\end{tabular}

${ }^{1}$ NLC: Acetylcholinesterase inhibitor (RVG)-loaded nanostructured lipid carriers; ${ }^{2}$ NanoE: nanoemulsion; ${ }^{3}$ In situ gel of RVG-loaded nanostructured lipid carriers; ${ }^{4}$ In situ gel of RVGloaded nanoemulsion.

From Table 1, it can be seen that the particle/droplet size of the RVG-loaded NLC and RVG-loaded nanoemulsion increased slightly after incorporation in the in situ gels. Similar results have been reported in other studies. For example, Fatouh et al. [14] observed a slight increase in the particle size of agomelatine-loaded SLN $(167.7 \pm 0.42 \mathrm{~nm})$ after incorporation in an in situ gel $(175.75 \pm 1.10 \mathrm{~nm})$, and Pires et al. [16] verified an increase in the droplet size of a fosphenytoin-loaded nanoemulsion $(216.4 \pm 10.5 \mathrm{~nm})$ after incorporation in a thermosensitive gel $(219.7 \pm 26.8 \mathrm{~nm})$.

\subsection{Organoleptic Analysis}

The organoleptic analysis of the formulations showed that the in situ gels were opaque and had a homogeneous appearance without visibly notable phase separation, before and after gelation.

\section{4. $\mathrm{pH}$ and Osmolarity Measurements}

The mean $\mathrm{pH}$ and osmolarity values obtained for the in situ gels of RVG-loaded NLC and the RVG-loaded nanoemulsion were $6.60 \pm 0.01$ and $275 \pm 0.02$ and $7.00 \pm 0.02$ and 280 $\pm 0.00 \mathrm{mOsm} / \mathrm{kg}$, respectively. Thus, the prepared formulations can be used for nasal application without damaging the nasal mucosa [17].

\subsection{Rheological and Texture Analysis}

Figures 1A,B show that the in situ gels of RVG-loaded NLC and RVG-loaded nanoemulsions exhibited a non-Newtonian plastic behavior, which means that viscosity decreases with increasing shear rate. Moreover, a decrease in the formulations' apparent viscosity with time was not observed, which indicates the absence of thixotropy (the upper and lower curves of the rheograms overlap). Other authors have reported the same behavior as desirable for nasal formulations [18-20]. Figure 1B also shows the effect of lipid-based nanosystems on the flow behavior. As expected, in situ gels of RVG-loaded NLC and RVG-loaded nanoemulsions had a lower viscosity than the in situ control gel, since lipid-based systems interfere with the consistency of the gels, decreasing their apparent viscosity.

Regarding the low viscosity of the in situ control gel with RVG before its gelation at $34.4 \pm 0.5{ }^{\circ} \mathrm{C}$, it is impossible to evaluate its flow behavior at $20.5 \pm 0.5^{\circ} \mathrm{C}$. 

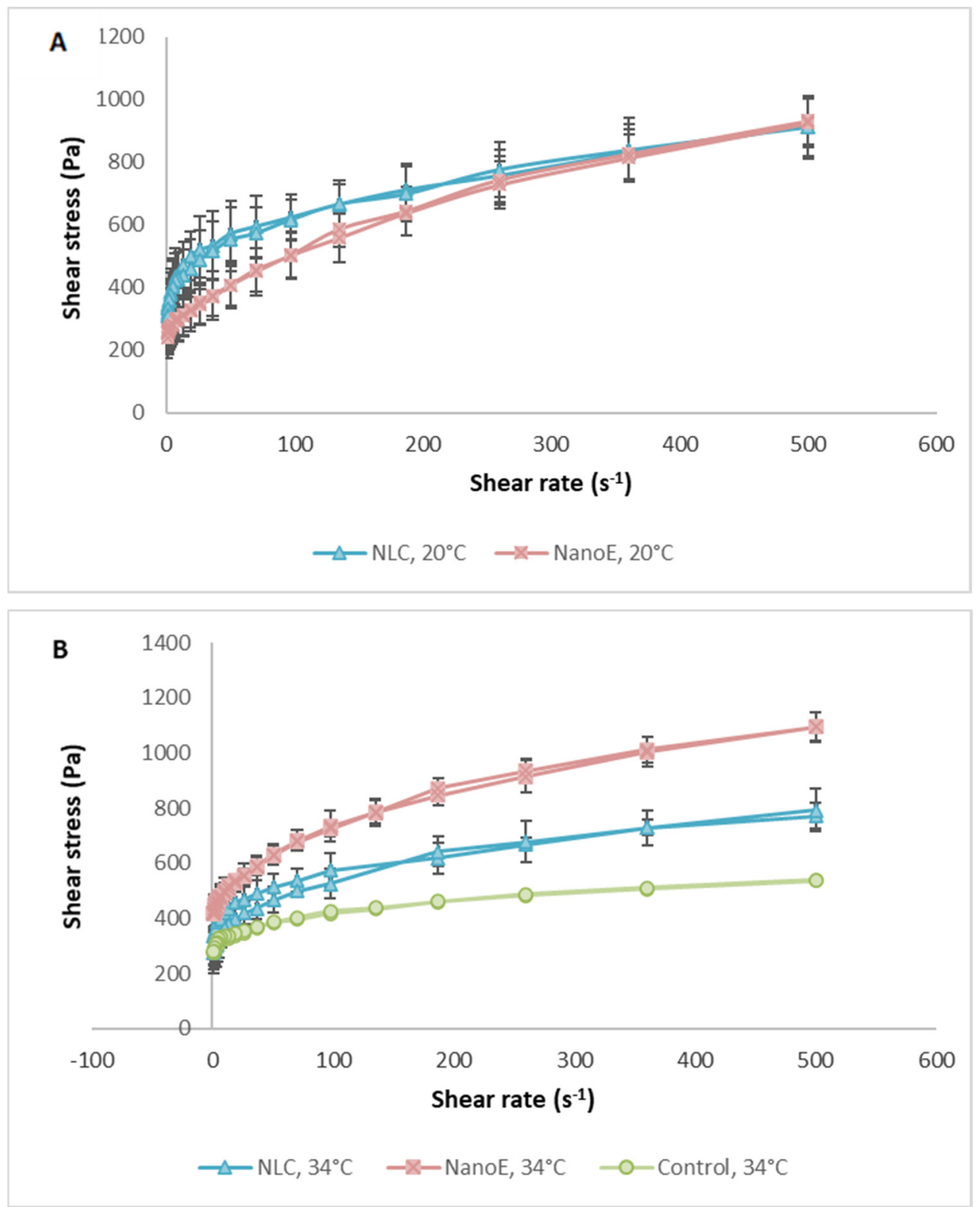

Figure 1. Rheological analysis of the in situ gel of RVG-loaded nanostructured lipid carriers (NLC) and in situ gel of RVG-loaded nanoemulsions, at $20.5 \pm 0.5^{\circ} \mathrm{C}(\mathbf{A})$ and $34.4 \pm 0.5^{\circ} \mathrm{C}(\mathbf{B})$.

Figures $2 \mathrm{~A}, \mathrm{~B}$ show the results of the texture analysis. Regarding the maximum force or firmness (Figure 2A), it was observed that the in situ gel of RVG-loaded NLC exhibited a higher firmness than the in situ gels of RVG-loaded nanoemulsions. This could be related to the lower viscosity of the nanoemulsions at $20.5 \pm 0.5^{\circ} \mathrm{C}$ than NLC. However, all formulations had higher values of firmness than the in situ control gel, meaning that the presence of the NLC and of the nanoemulsions increases the firmness of the in situ gels. Concerning the adhesiveness (Figure 2B), the in situ gel of RVG-loaded NLCalso exhibits higher values of adhesion $(-0.945$ N.mm and -1.041 N.mm, respectively) than the in situ gel of RVG-loaded nanoemulsions $(-0.625 \mathrm{~N} . \mathrm{mm})$. In addition, both formulations presented a higher adhesion than the in situ control gel $(-0.4863 \mathrm{~N} . \mathrm{mm})$. These results suggest that the in situ gels of lipid-based nanosystems increase the formulations' retention time in the nasal cavity [21]. 

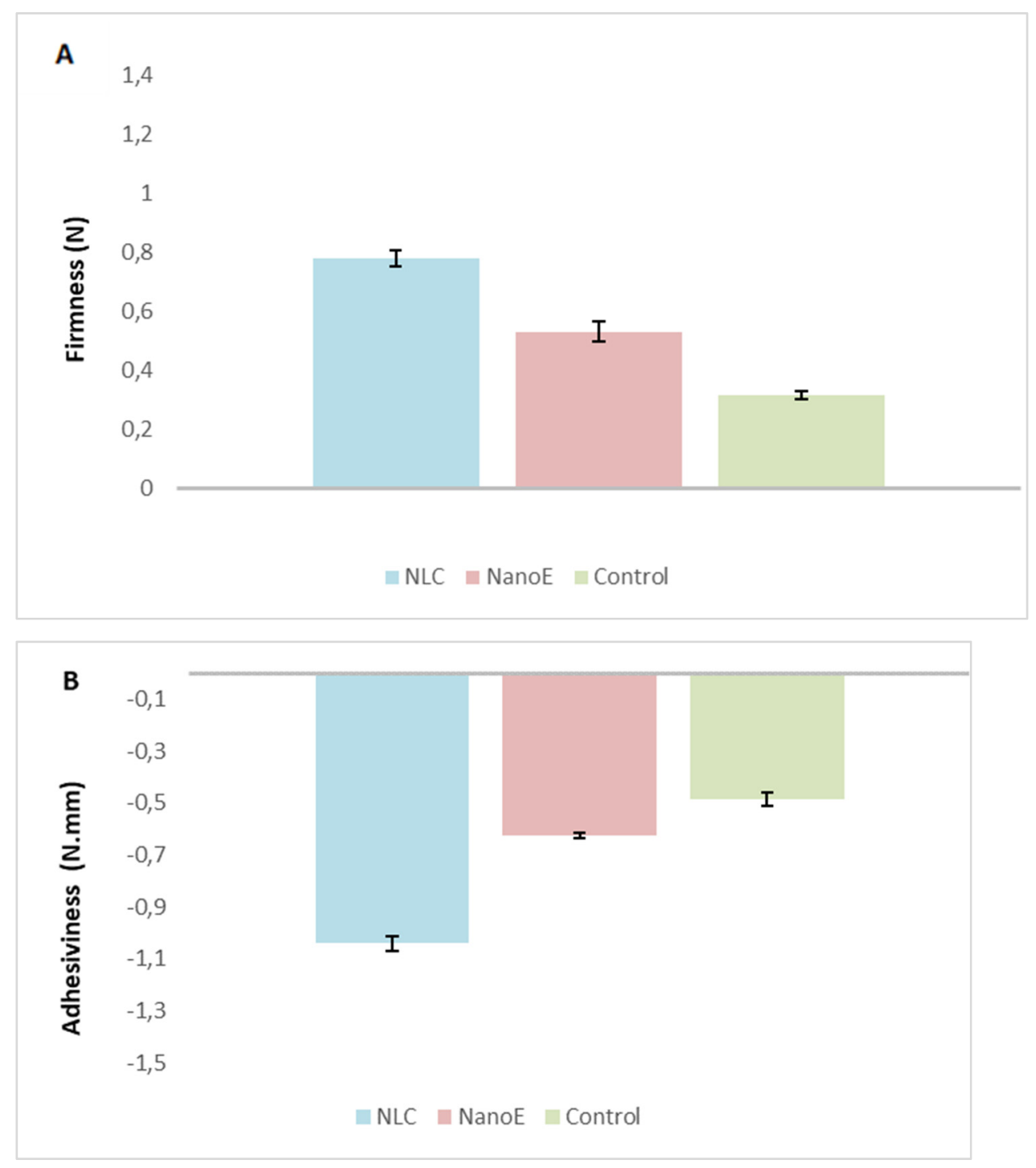

Figure 2. Texture analysis of the in situ gel of RVG-loaded NLC, in situ gel of RVG-loaded nanoemulsion, and in situ control gel, measured at $20.5 \pm 0.5^{\circ} \mathrm{C}$. (A) Firmness; (B) adhesiveness.

\section{Conclusions}

The developed thermosensitive in situ gels of RVG-loaded NLC and of RVG-loaded nanoemulsions had adequate values of particle/droplet size, PDI, and ZP for nasal administration. They also presented a homogeneous appearance and values of $\mathrm{pH}$ and osmolarity compatible with the physiological values of the nasal cavity. Rheological studies demonstrated that both of the thermosensitive in situ gels exhibited a plastic non-Newtonian behavior without thixotropy. Regarding the texture analysis, it was observed that the addition of thermosensitive and mucoadhesive polymers to the RVG-loaded NLC and RVG-loaded nanoemulsions increased their firmness and adhesiveness. From these results, we conclude that the developed thermosensitive in situ gels can be used to improve the treatment of $\mathrm{AD}$ through the nose-to-brain route. However, this study is under development, and further in vitro and ex vivo studies should be performed to confirm this application.

Author Contributions: A.C.S. and S.C. conceived and designed the experiments; S.C. performed the experiments; A.C.S. and S.C. analyzed the data; S.C. wrote the paper; A.C.S., B.F. and J.M.S.L. revised the paper. All authors have read and agreed to the published version of the manuscript.

Institutional Review Board Statement: Not applicable. 
Informed Consent Statement: Not applicable.

Data Availability Statement: Data is contained within the article. The data presented in this study are available in [Cunha, S., Forbes, B., Lobo, J. M. S., \& Silva, A. C. 2020. Thermosensitive Nasal In Situ Gels of Lipid-Based Nanosystems to Improve the Treatment of Alzheimer's Disease; In Presented at the 1st International Electronic Conference on Pharmaceutics; Vol. 1, p. 15.

Acknowledgments: This work was supported by Fundação para a Ciência e a Tecnologia (FCT), Portugal (SFRH/131074/2017), by the Applied Molecular Biosciences Unit-UCIBIO (UID/Multi/04378/2019), FP-ENAS (UID/Multi/04546/2019).

Conflicts of Interest: The authors declare no conflict of interest.

\section{Abbreviations}

The following abbreviations are used in this manuscript:

$\begin{array}{ll}\text { AD } & \text { Alzheimer's disease } \\ \text { CNS } & \text { Central nervous system } \\ \text { BBB } & \text { Blood-brain barrier } \\ \text { NLC } & \text { Nanostructured lipid carriers } \\ \text { NanoE } & \text { Nanoemulsions } \\ \text { RVG } & \text { Anticholinesterase drug } \\ \text { PDI } & \text { Polydispersity index } \\ \text { ZP } & \text { Zeta potential }\end{array}$

\section{References}

1. Kales, H.C.; Lyketsos, C.G.; Miller, E.M.; Ballard, C. Management of behavioral and psychological symptoms in people with Alzheimer's disease: An international Delphi consensus. Int. Psychogeriatr. 2019, 31, 83-90.

2. Atri, A. Current and Future Treatments in Alzheimer's Disease. Semin. Neurol. 2019, 39, 227-240, doi:10.1055/s-0039-1678581.

3. Sharma, P.; Tripathi, M.K.; Shrivastava, S.K. Cholinesterase as a Target for Drug Development in Alzheimer's Disease. In Targeting Enzymes for Pharmaceutical Development; Springer: Berlin/Heidelberg, Germany, 2020; pp. 257-286.

4. Sweeney, M.D.; Zhao, Z.; Montagne, A.; Nelson, A.R.; Zlokovic, B.V. Blood-brain barrier: From physiology to disease and back. Physiol. Rev. 2019, 99, 21-78.

5. Cunha, S.; Amaral, M.; Lobo, J.S.; Silva, A.C. Lipid nanoparticles for nasal/intranasal drug delivery. Crit. Rev. Ther. Drug Carr. Syst. 2017, 34, 257-282.

6. Cunha, S.; Almeida, H.; Amaral, M.; Lobo, J.S.; Silva, A. Intranasal lipid nanoparticles for the treatment of neurodegenerative diseases. Curr. Pharm. Des. 2017, 23, 6553-6562.

7. Costa, C.; Moreira, J.; Amaral, M.; Lobo, J.S.; Silva, A. Nose-to-brain delivery of lipid-based nanosystems for epileptic seizures and anxiety crisis. J. Control. Release 2019, 295, 187-200.

8. Makhlouf, A.S.H.; Abu-Thabit, N.Y. Advances in Smart Coatings and Thin Films for Future Industrial and Biomedical Engineering Applications; Elsevier: Amsterdam, The Netherlands, 2019.

9. Sabale, A.S.; Kulkarni, A.D.; Sabale, A.S. Nasal In Situ Gel: Novel Approach for Nasal Drug Delivery. J. Drug Deliv. Ther. 2020, 10, 183-197.

10. Jiang, T.; Liao, W.; Charcosset, C. Recent advances in encapsulation of curcumin in nanoemulsions: A review of encapsulation technologies, bioaccessibility and applications. Food Res. Int. 2020, 132, 109035.

11. Gadhave, D.G.; Kokare, C.R. Nanostructured lipid carriers engineered for intranasal delivery of teriflunomide in multiple sclerosis: Optimization and in vivo studies. Drug Dev. Ind. Pharm. 2019, 45, 839-851.

12. Cunha, S.; Costa, C.P.; Loureiro, J.A.; Alves, J.; Peixoto, A.F.; Forbes, B.; Sousa Lobo, J.M.; Silva, A.C. Double Optimization of Rivastigmine-Loaded Nanostructured Lipid Carriers (NLC) for Nose-to-Brain Delivery Using the Quality by Design (QbD) Approach: Formulation Variables and Instrumental Parameters. Pharmaceutics 2020, 12, 599.

13. Wang, Q.; Zuo, Z.; Cheung, C.K.C.; Leung, S.S.Y. Updates on thermosensitive hydrogel for nasal, ocular and cutaneous delivery. Int. J. Pharm. 2019, 559, 86-101.

14. Fatouh, A.M.; Elshafeey, A.H.; Abdelbary, A. Agomelatine-based in situ gels for brain targeting via the nasal route: Statistical optimization, in vitro, and in vivo evaluation. Drug Deliv. 2017, 24, 1077-1085.

15. Almeida, H.; Helena Amaral, M.; Lobão, P.; Manuel Sousa Lobo, J. Influence of drug incorporation, temperature and storage time on the $\mathrm{pH}$, textural and rheological properties of different poloxamer hydrogels. Curr. Drug Deliv. 2013, 10, 753-764.

16. Pires, P.C.; Peixoto, D.; Teixeira, I.; Rodrigues, M.; Alves, G.; Santos, A.O. Nanoemulsions and thermosensitive nanoemulgels of phenytoin and fosphenytoin for intranasal administration: Formulation development and in vitro characterization. Eur. J. Pharm. Sci. 2020, 141, 105099.

17. Scherließ, R. Nasal formulations for drug administration and characterization of nasal preparations in drug delivery. Ther. Deliv. 2020, 11, 183-191. 
18. Gholizadeh, H.; Cheng, S.; Pozzoli, M.; Messerotti, E.; Traini, D.; Young, P.; Kourmatzis, A.; Ong, H.X. Smart thermosensitive chitosan hydrogel for nasal delivery of ibuprofen to treat neurological disorders. Expert Opin. Drug Deliv. 2019, 16, 453-466.

19. Adnet, T.; Groo, A.-C.; Picard, C.; Davis, A.; Corvaisier, S.; Since, M.; Bounoure, F.; Rochais, C.; Pluart, L.L.; Dallemagne, P. Pharmacotechnical Development of a Nasal Drug Delivery Composite Nanosystem Intended for Alzheimer's Disease Treatment. Pharmaceutics 2020, 12, 251.

20. Soliman, M.E.; Elmowafy, E.; Casettari, L.; Alexander, C. Star-shaped poly (oligoethylene glycol) copolymer-based gels: Thermo-responsive behaviour and bioapplicability for risedronate intranasal delivery. Int. J. Pharm. 2018, 543, $224-233$.

21. Basu, S.; Bandyopadhyay, A.K. Development and characterization of mucoadhesive in situ nasal gel of midazolam prepared with Ficus carica mucilage. AAPS Pharmscitech 2010, 11, 1223-1231. 